\title{
A CONTINUOUS OPTIMIZATION MODEL FOR A JOINT PROBLEM OF PRICING AND RESOURCE ALLOCATION
}

\author{
Mustapha Bouhtou $^{1}$ and Guillaume Erbs ${ }^{1,2}$
}

\begin{abstract}
This paper investigates the problem of maximizing the revenue of a telecommunications operator by simultaneously pricing point-to-point services and allocating bandwidth in its network, while facing competition. Customers are distributed into market segments, i.e., groups of customers with a similar preference for the services. This preference is expressed using utility functions, and customers choose between the offers of the operator and of the competition according to their utility. We model the problem as a leader-follower game between the operator and the customers. This kind of problem has classically been modeled as a bilevel program. A market segmentation is usually defined by a discrete distribution function of the total demand for a service; in this case, the problem can be modeled as a combinatorial optimization problem. In this paper, however, we motivate the use of a continuous distribution function and investigate the nonlinear continuous optimization problem obtained in this case. We analyze the mathematical properties of the problem, and in particular we give a necessary and sufficient condition for its convexity. We introduce methods to solve the problem and we provide encouraging numerical results on realistic telecommunications instances of the problem, showing that it can be solved efficiently.
\end{abstract}

Keywords. Optimization, bilevel programming, revenue management, pricing, resource allocation, telecommunications.

Mathematics Subject Classification. 90C90.

Received April 2, 2007. Accepted March 27, 2008.

1 France Telecom Research and Development, 38-40 rue du Général Leclerc, 92794 Issy les Moulineaux, France; mustapha.bouhtou@orange-ftgroup.com

2 Université Paris 6, 4 place Jussieu, 75005 Paris, France; guillaume.erbs@gdfsuez.com 


\section{INTRODUCTION}

Revenue management techniques have had such a successful application in the airline industry that various other industries have been borrowing ideas to apply to their field. For example, there were successful adaptations to car rental or hotels (e.g., see McGill and van Ryzin [23] for more details).

Lately, various applications to the telecommunications industry have been proposed. Nair and Bapna [24] defined an application for Internet Service Providers to manage their revenue by controlling the connections to their modem pool so that quality of service is ensured to their highest paying customers. Keon and Anandalingam [18] proposed a dynamic pricing scheme for multi-service networks by offering a discount as an incentive for a customer to shift its demand from a congested period to another (less congested) period. In [17] they also proposed a pricing and resource allocation model to guarantee quality of service in a multi-service network. Bouhtou et al. [4,5] introduced a framework to optimize telecommunication link prices using shadow pricing. Bouhtou et al. [8,15] studied a bilevel model for pricing arcs in a network.

In this paper, we consider a telecommunications operator willing to allocate bandwidth in its network based on the revenue generated by the services it sells, while taking into account the competition. The telecommunications services provided, which use the available bandwidth, are defined by their price and their characteristics (data rate, network type, etc.). For example, these services could be leased lines or virtual private networks. In order to take into account the competition, we model the behavior of the customers in the market by their utility for a service. Using statistical tools, the customers can be classified in various groups having similar needs and behavior, called market segments. The operator wants to define the price of its offers on each of the targeted markets and determine the bandwidth to allocate to each offer in its network. The customers want to get the best offer, choosing between the operator and the competitors, according to their preferences. A model for this problem was introduced by Côté et al. [11] in the context of the airline industry. Bouhtou et al. [6,7] extended it to the telecommunications industry. Fortin et al. [13] also proposed a model for dealing with congestion in the network, which is also a way of modelling capacity.

This kind of problems, a Stackelberg game, has traditionally been modeled as a bilevel program (see for instance Labbé et al. [19,20], Brotcorne et al. [9,10], Côté et al. [11]). Bouhtou et al. [7] showed that in the case of one offer on each market it can be reformulated as a non-concave resource allocation whose objective function is continuous and piecewise linear. In this formulation, the decision variables are the capacities, and the prices are computed implicitly. The problem results in finding which market segments to target on each market and allocating resources accordingly. The model can then solved efficiently using mixed integer linear programming techniques. 
An issue in the studies cited before is that the market segmentation is supposed to be discrete, that is the demand of each market is distributed over a discrete number of market segments. This implies an unrealistic behavior of the clients: they choose to buy (or not) bandwidth according to the price and thus a small change in the price of a product can make an entire market segment choose the competition, while in reality not all the customers of a market segment have exactly the same utility. One way of dealing with such a behavior would be to use a random utility model, which leads to the use of discrete choice models (e.g., see Ben-Akiva and Bierlaire [2] for the use of such models in transportation). This leads to interesting and difficult problems [14], but this is out of the scope of this paper. Another issue is the lack of precision of the data resulting from the statistical studies that give the market segmentation; indeed, this data gives a discrete distribution function, while a continuous distribution function might give a better approximation of the real situation (although it might not be as intuitively interpreted). In this paper, we address these problems by studying the case of a continuous demand distribution. Moreover, there is a mathematical interest in using continuous distributions. In the discrete case, the problem is combinatorial, while in the continuous case, we show that under some conditions the problem is convex, and thus "easy" to solve using nonlinear solvers.

A continuous model for classes of customers was previously used by Marcotte and Zhu [22] to model traffic equilibria. Marcotte et al. [21] also recently studied a continuous model for a bilevel programming approach to a Stackelberg game. The context of their work is a problem of pricing the legs of a flight. They consider a continuous market segmentation and study the mathematical properties of the bilevel programming model they obtain. They propose a descent method to solve the problem. Our study differs on two aspects: our model sets a price on a path of the network, and not its arcs; furthermore, we use a different modeling approach for a similar problem.

The objective of this paper is to study the feasibility of solving this problem using a continuous demand distribution. Our contribution is a mathematical study of the model that leads to a necessary and sufficient condition on the distribution function for convexity of the problem. Using a distribution function satisfying this condition, we also provide numerical experiments on realistic networks connecting major French cities.

This paper is organized as follows. In Section 2 the problem is introduced. In Section 3, two bilevel programming models are given for the problem, one with a continuous demand distribution, the other with a discrete demand distribution. In Section 4, we recall two solving methods for the discrete case, using mixed integer formulations of the problem $[6,7]$. In Section 5, the resource allocation formulation [7] is adapted to the continuous case. Mathematical properties of the objective function in this case are given, and a necessary and sufficient condition for convexity of the problem is obtained. In Section 6, the Weibull distribution, which satisfies the necessary and sufficient condition, is studied as an example. In Section 7, algorithmic aspects are presented. A solution method for the continuous 
case using the Weibull function is given. Finally, in Section 7.3, numerical results and comparisons between both models are presented.

\section{The JoInt PRICING AND RESOURCE ALLOCATION PROBLEM}

The problem we study here was largely introduced in [7]. It considers the optimization of the revenue of an operator who, using its own network, wants to provide competitive offers for point to point telecommunication services in a set of markets where the competition is active. A market is defined by one origindestination pair in the network.

In each market, the operator and the competition have one offer each (thus, we will refer indifferently to markets or offers). With each offer is associated one path on the network along which the traffic of the clients is sent. Each offer $k$ we consider is characterized by two parameters: its tariff $T_{k}$ and an aggregated value of its features $D_{k}$ (data rate, type of network, network availability and reliability, etc.). For the competition, these parameters are assumed to be publicly available, and they are static, i.e., the competition does not change its offers when the operator sets its tariffs. We suppose that the arc capacities in the operator network are limited. If there is not enough capacity to meet the total demand, we assume that a competitor will want to offer services on this market. This implies that the competition can be supposed to have infinite capacity on its network. The clients have different perceptions of the services offered. Hence, groups of customers with similar needs and perceptions of the offers can be identified, and form what we refer to as market segments. In our model, to define how valuable the features of an offer are for a segment $s$, a unit price $\alpha_{s}$ is used and a money valuation is introduced as a linear function: $\alpha_{s} \times D_{k}$.

To model the perception of the global cost of an offer by the clients, the "apparent price" concept is used. It is a compromise between the nominal price (tariff of the offer) and the client's money valuation of the features of the offer. Our apparent price model is simply obtained by adding the nominal tariff to the client's money valuation function. Hence, the clients make their decision according to the apparent price of each offer, and choose the lowest one. We assume that the clients can split their demand between the operator and the competition. This might happen if the operator provides a lowest apparent price but has not enough capacity to satisfy the demand. We also suppose that we have a forecast of the demand for each market, and a distribution function of this demand over the market segments.

In order to model the problem, we represent the telecommunication network of the operator as a directed graph $N=(V, \mathcal{A})$ with a transmission capacity on each $\operatorname{arc} ; \mathcal{A}$ is the set of indices of the arcs of the network. We denote by $\mathcal{M}$ the set of indices of the markets and $\mathcal{S}$ the set of indices of the market segments. 
The input data defining the problem are:

$d_{k} \quad$ total demand of all clients on market $k$;

$\phi_{k}(s) \quad$ demand of segment $s$ on market $k$;

$\phi_{k}($.$) is the distribution function of the demand on market k$;

$T_{k}^{c} \quad$ competition tariff on market $k$;

$\bar{T}_{k} \quad$ maximum tariff that can be set on market $k$ (supposed greater than $\left.T_{k}^{c}\right)$

$D_{k} \quad$ value of features of the operator offer on market $k$;

$D_{k}^{c} \quad$ value of features of the competition offer on market $k$;

$\alpha_{s} \quad$ unit price for the features of an offer associated with a segment $s$,

$\Pi_{s}(T, D)$ apparent price function of segment $s$;

where $T$ is the tariff of the offer and $D$ the value of its features;

$\Pi_{s}(T, D)=T+\alpha_{s} \times D$;

$\mathcal{A}(k) \quad$ routing path used for the offer $k$ of the operator;

$c_{a} \quad$ resource capacity of $\operatorname{arc} a$ in the network.

Before introducing the model, let $v_{k}^{1}(s)$ (resp. $v_{k}^{2}(s)$ ) be the traffic which will be induced by segment $s$ on the path corresponding to the offer of the operator (resp. the competition) on market $k$ (one must have $v_{k}^{1}(s)+v_{k}^{2}(s)=\phi_{k}(s)$ ). Let $T_{k}$ denotes the operator tariff to set on market $k$ and $q_{k}$ denotes the amount of capacity to allocate to the routing path used on market $k$. The components $v_{k}^{1}(s), v_{k}^{2}(s), T_{k}$ and $q_{k}$ are the main variables of the optimization problem we will deal with.

\section{Bilevel optimization models}

The problem is a Stackelberg game that is classically modeled using a bilevel program (Labbé et al. [19,20], Brotcorne et al. [9,10], Côté et al. [11]). Two similar models can be written, using a continuous demand distribution, or by discretizing this distribution.

\subsection{Model Based on a Continuous Demand distribution}

To define a continuous model, without loss of generality, we suppose the set of the segments $\mathcal{S}$ is represented by the interval $[0,+\infty[$ in the real set $\mathbb{R}$ and we will confuse each $s$ in $\mathcal{S}$ with $\alpha_{s}$ its unit price valuation related to the features of an offer (i.e., $\alpha_{s}=s$ ). We also suppose that the demand distribution $\phi_{k}($.$) is a$ positive continuous function. In this case, the joint pricing and allocation resource optimization model is given as follows:

$$
\begin{aligned}
& \left.\operatorname{P}_{c}\right) \max _{T_{k}, v_{k}^{1}(s), q_{k}} \sum_{k \in \mathcal{M}} T_{k} \int_{s \in \mathcal{S}} v_{k}^{1}(s) \mathrm{d} s \\
\text { s.t. } & \sum_{k \in \mathcal{M} \mid a \in \mathcal{A}(k)} q_{k} \leq c_{a} \quad \forall a \in \mathcal{A}
\end{aligned}
$$




$$
\begin{aligned}
& \bar{T}_{k} \geq T_{k} \geq 0 \quad \forall k \in \mathcal{M} \\
& \min _{v_{k}^{1}(s), v_{k}^{2}(s)} \sum_{k \in \mathcal{M}} \int_{s \in \mathcal{S}} \Pi_{s}\left(T_{k}, D_{k}\right) v_{k}^{1}(s) \mathrm{d} s+\sum_{k \in \mathcal{M}} \int_{s \in \mathcal{S}} \Pi_{s}\left(T_{k}^{c}, D_{k}^{c}\right) v_{k}^{2}(s) \mathrm{d} s \\
& \text { s.t. } v_{k}^{1}(s)+v_{k}^{2}(s)=\phi_{k}(s) \quad \forall k \in \mathcal{M}, \quad \forall s \in \mathcal{S} . \\
& \int_{s \in \mathcal{S}} v_{k}^{1}(s) \mathrm{d} s \leq q_{k} \quad \forall k \in \mathcal{M} \\
& v_{k}^{1}(s), v_{k}^{2}(s) \geq 0 \quad \forall k \in \mathcal{M}, \forall s \in \mathcal{S}
\end{aligned}
$$

From the operator's point of view, the objective is to maximize its revenue (Eq. (1)) while allocating capacities for each service that do not exceed physical (or already pre-allocated) capacities of each link (constraint (2)). The tariffs are non-negative and bounded (constraint (3)).

From the clients' point of view, the objective is to minimize the price they pay to either the operator or the competition (Eq. (4)), considering the apparent prices corresponding to the features offered. The clients want all their demand to be satisfied (constraint (5)), while it can only be met up to the allocated capacity on the operator's network (constraint (6)). The traffic is non-negative (constraint (7)).

\subsection{Model Based on a discrete Demand Distribution}

By sampling the demand distribution, one can obtain a discrete model for the problem:

$$
\begin{array}{lll}
\left(P_{d}\right) \max _{T_{k}, v_{k}^{1}(s)} & \sum_{k \in \mathcal{M}} T_{k} \sum_{s \in \mathcal{S}} v_{k}^{1}(s) & \\
\text { s.t. } & \sum_{k \in \mathcal{M} \mid a \in \mathcal{A}(k)} q_{k} \leq c_{a} & \forall a \in \mathcal{A} \\
& \bar{T}_{k} \geq T_{k} \geq 0 & \forall k \in \mathcal{M} \\
\min _{v_{k}^{1}(s), v_{k}^{2}(s)} & \sum_{k \in \mathcal{M}} \sum_{s \in \mathcal{S}} \Pi_{s}\left(T_{k}, D_{k}\right) v_{k}^{1}(s)+\Pi_{s}\left(T_{k}^{c}, D_{k}^{c}\right) v_{k}^{2}(s) & \\
\text { s.t. } & v_{k}^{1}(s)+v_{k}^{2}(s)=\phi_{k}(s) & \forall k \in \mathcal{M}, \forall s \in \mathcal{S} \\
& \sum_{s \in \mathcal{S}} v_{k}^{1}(s) \leq q_{k} & \forall k \in \mathcal{M} \\
& v_{k}^{1}(s), v_{k}^{2}(s) \geq 0 & \forall k \in \mathcal{M}, \forall s \in \mathcal{S} .
\end{array}
$$

\section{Solving The MOdel BASED on A Discrete Demand DISTRIBUTION}

\subsection{Classical approach}

As shown for instance in $[6,9,10,19,20]$, using the Karush-Kuhn-Tucker conditions for the lower level linear program, the problem can be reformulated as 
a single level nonlinear mathematical program with complementarity constraints. The complementarity constraints can be linearized using binary variables and "big M" constants in order to obtain a mixed integer linear program (MILP). Using this approach, Côté et al. [11] made experiments on airline networks; Bouhtou et al. [6] made similar experiments on telecommunications networks. With both types of networks, this approach is able to solve only small instances. The solver cannot handle the large number of binary variables that this problem induces. Another difficulty is induced by the capacity constraints, that couple the markets. On a telecommunications network, many routes can share a link, and thus this makes the problem highly combinatorial in nature.

\subsection{Approach BASED on A ReSOURCE ALlocAtion MOdel}

To address the main difficulties due to the MILP approach to the bilevel program, Bouhtou et al. [7] introduce an alternative approach where the optimization problem is considered as a resource allocation problem with a particular objective function. In this new model, the decision variables are restricted to the allocated capacities $q_{k}$ for each offer while the tariff variables are handled implicitly. As shown in [7], the problem $\left(P_{d}\right)$ can be reformulated as follows:

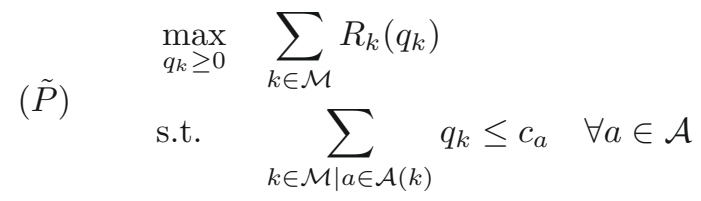

where $R_{k}\left(q_{k}\right)$ is the optimal value of the revenue that could be provided by the market $k$ when its allocation capacity is set to $q_{k}$; it is given by:

$$
\begin{array}{cc}
R_{k}\left(q_{k}\right)=\max _{T_{k}, v_{k}^{1}(s)} & T_{k} \sum_{s \in \mathcal{S}} v_{k}^{1}(s) \\
\text { s.t. } & \bar{T}_{k} \geq T_{k} \geq 0
\end{array}
$$

$$
\begin{array}{lll}
\min _{v_{k}^{1}(s), v_{k}^{2}(s)} & \sum_{s \in \mathcal{S}} \Pi_{s}\left(T_{k}, D_{k}\right) v_{k}^{1}(s)+\sum_{s \in \mathcal{S}} \Pi_{s}\left(T_{k}^{c}, D_{k}^{c}\right) v_{k}^{2}(s) & \\
\text { s.t. } & v_{k}^{1}(s)+v_{k}^{2}(s)=\phi_{k}(s) & \\
& \sum_{s \in \mathcal{S}} v_{k}^{1}(s) \leq q_{k} & \\
& v_{k}^{1}(s), v_{k}^{2}(s) \geq 0 & \forall s \in \mathcal{S} .
\end{array}
$$

In [7], it is shown that in the discrete case the value function $R_{k}($.$) is well defined$ as a continuous, non-decreasing, piecewise linear function, with alternating linear and constant pieces (see Fig. 1). 


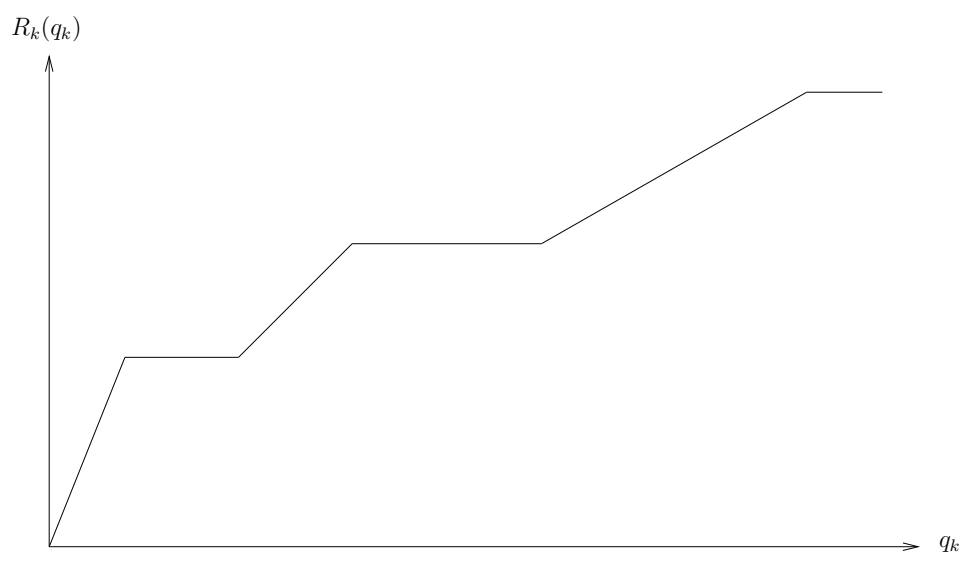

FiguRE 1. Example of an objective function $R_{k}\left(q_{k}\right)$.

In [7], this model is also formulated as a MILP, using the so-called multiple choice formulation: binary variables are introduced in order to select the linear pieces of the function on each market, and one must choose exactly one of the linear pieces for each market.

This MILP was solved using the branch-and-cut algorithm from CPLEX. The results obtained and reported in [7] show great improvements compared to the MILP approach for the bilevel model, and optimal solutions to instances that could not be solved before are found.

\section{Study of the MOdel With A CONTINUOUS DEMAND DISTRIBUTION FUNCTION}

In the case where the demand distribution functions $\phi_{k}($.$) are continuous, as$ in the previous section, the problem can also be reduced to a resource allocation optimization model.

The same optimization problem formulation $(\tilde{P})$

$$
\begin{array}{ll}
\max _{q_{k} \geq 0} & \sum_{k \in \mathcal{M}} R_{k}\left(q_{k}\right) \\
\text { s.t. } & \sum_{k \in \mathcal{M} \mid a \in \mathcal{A}(k)} q_{k} \leq c_{a} \quad \forall a \in \mathcal{A}
\end{array}
$$


can naturally be used but the objective components $R_{k}\left(q_{k}\right)$ are defined by

$$
\begin{array}{ll}
R_{k}\left(q_{k}\right)=\max _{T_{k}, v_{k}^{1}(s)} & T_{k} \int_{s \in \mathcal{S}} v_{k}^{1}(s) \mathrm{d} s \\
\text { s.t. } & \bar{T}_{k} \geq T_{k} \geq 0 \\
\min _{v_{k}^{1}(s), v_{k}^{2}(s)} & \int_{s \in \mathcal{S}} \Pi_{s}\left(T_{k}, D_{k}\right) v_{k}^{1}(s) \mathrm{d} s+\int_{s \in \mathcal{S}} \Pi_{s}\left(T_{k}^{c}, D_{k}^{c}\right) v_{k}^{2}(s) \mathrm{d} s \\
\text { s.t. } & v_{k}^{1}(s)+v_{k}^{2}(s)=\phi_{k}(s) \\
& \int_{s \in \mathcal{S}} v_{k}^{1}(s) \mathrm{d} s \leq q_{k} \\
& v_{k}^{1}(s), v_{k}^{2}(s) \geq 0
\end{array}
$$

In this case, the optimization model $(\tilde{P})$ obtained belongs to a set of nonlinear continuous optimization problems: the objective is continuous but nonlinear and the constraints are linear. Also note that the objective to maximize remains non-smooth since its components $R_{k}\left(q_{k}\right)$ represent value functions of optimization problems which are non-smooth in general. In this section we will analyze some mathematical properties of this nonlinear model and deduce conditions under which the optimization problem may be easy to solve. In particular, we will give the necessary and sufficient convexity conditions of the problem.

We introduce the following notation:

- $a_{k}=D_{k}^{c}-D_{k}$ the gap between the values of the features offered by the operator and the competition on market $k$;

- $\Gamma_{k}(T)=\left\{\alpha \geq 0 / T \leq T_{k}^{c}+\alpha \times a_{k}\right\}$ the set of segments the operator could capture on market $k$ when setting the tariff to $T$;

- $\bar{q}_{k}=\int_{\alpha \in \Gamma_{k}(0)} \phi_{k}(\alpha) \mathrm{d} \alpha$ the potential maximum demand the operator could capture on market $k$ when the tariff is set to zero;

- $\underline{q}_{k}=\int_{\alpha \in \Gamma_{k}\left(\bar{T}_{k}\right)} \phi_{k}(\alpha) \mathrm{d} \alpha$ the potential maximum demand the operator could capture on market $k$ when the tariff is set to $\bar{T}_{k}$;

- $R_{k}(q, T)$ the revenue value on market $k$ when the capacity allocation and the tariff are respectively set to $q$ and $T$;

- $\bar{\alpha}_{k}=\left\{\begin{array}{ll}\frac{\bar{T}_{k}-T_{k}^{c}}{a_{k}} & \text { if } a_{k}>0 \\ -\frac{T^{c}}{a_{k}} & \text { if } a_{k}<0\end{array}\right.$ this parameter gives an upper bound on the set

$$
\left\{\alpha \geq 0: 0 \leq T_{k}^{c}+\alpha a_{k} \leq \bar{T}_{k}\right\} .
$$

Remark 5.1. In the case where $a_{k}=0$ on market $k$, it is easy to see that an optimal tariff is simply given by $T_{k}=T_{k}^{c}$. Thus, we will not consider this trivial case and then, in what follows, we will assume that $\forall k, a_{k} \neq 0$.

Using the definition of $R_{k}(q)$, one can easily see that:

$$
R_{k}(q)=\max _{\bar{T}_{k} \geq T \geq 0} R_{k}(q, T) .
$$


Lemma 5.1. If $T_{k}^{*}$ is an optimal value of the tariff on market $k$ such that $\Gamma_{k}\left(T_{k}^{*}\right)$ is not empty then there exists $\alpha_{k}>0$ such that $T_{k}^{*}=T_{k}^{c}+\alpha_{k} \times a_{k}$.

Proof. Assume that $\Gamma_{k}(T)$ is not empty. Hence, if $a_{k}<0$, we have $T_{k}^{*} \leq T_{k}^{c}$ and then $\exists \alpha_{k}>0$ such that $T_{k}^{*}=T_{k}^{c}+\alpha_{k} \times a_{k}$.

Let $q_{k}^{*}$ be the optimal allocated capacity. If $a_{k}>0$, we necessarily have $T_{k}^{*} \geq T_{k}^{c}$; otherwise, one can easily see that $\left(T_{k}^{c}, q_{k}^{*}\right)$ would remain a feasible solution and would provide more revenue. Finally, $a_{k}>0$ and $T_{k}^{*} \geq T_{k}^{c}$ ensure that $\exists \alpha_{k}>0$ such that $T_{k}^{*}=T_{k}^{c}+\alpha_{k} \times a_{k}$.

The previous result means that one may work directly with variables $\alpha$ instead of tariff variables; hence, the following result is immediate.

\section{Lemma 5.2.}

$$
R_{k}(q)=\max _{\bar{\alpha}_{k} \geq \alpha \geq 0} R_{k}\left(q, T_{k}^{c}+\alpha \times a_{k}\right) .
$$

The next result gives more specification about $R_{k}(q, T)$.

Lemma 5.3. The revenue function $R_{k}(q, T)$ verifies:

(1) $R_{k}(q, T)=T q$ if $q \leq \int_{\gamma \in \Gamma_{k}(T)} \phi_{k}(\gamma) \mathrm{d} \gamma$;

(2) $R_{k}(q, T)=T \int_{\gamma \in \Gamma_{k}(T)} \phi_{k}(\gamma) \mathrm{d} \gamma$ otherwise.

Proof. Let $\left(v_{k}^{1}(\gamma), v_{k}^{2}(\gamma)\right)$ be an optimal solution of the clients minimization problem (lower level). Seeing that $v_{k}^{2}(\gamma)=\phi_{k}(\gamma)-v_{k}^{1}(\gamma)$, the clients optimization problem is actually just a linear maximization problem on variables $v_{k}^{1}(\gamma)$.

Therefore, if $q \leq \int_{\gamma \in \Gamma_{k}(T)} \phi_{k}(\gamma) \mathrm{d} \gamma$, we have

$$
\int_{\gamma \in \Gamma_{k}(T)} v_{k}^{1}(\gamma) \mathrm{d} \gamma \leq q \leq \int_{\gamma \in \Gamma(T)} \phi_{k}(\gamma) \mathrm{d} \gamma
$$

and the maximization necessarily gives:

$$
q=\int_{\gamma \in \Gamma_{k}(T)} v_{k}^{1}(\gamma) \mathrm{d} \gamma
$$

Similarly, if $q>\int_{\gamma \in \Gamma_{k}(T)} \phi_{k}(\gamma) \mathrm{d} \gamma$, we have

$$
\int_{\gamma \in \Gamma_{k}(T)} v_{k}^{1}(\gamma) \mathrm{d} \gamma \leq \int_{\gamma \in \Gamma_{k}(T)} \phi_{k}(\gamma) \mathrm{d} \gamma<q
$$

and again the maximization necessarily gives:

$$
\int_{\gamma \in \Gamma_{k}(T)} v_{k}^{1}(\gamma) \mathrm{d} \gamma=\int_{\gamma \in \Gamma(T)} \phi_{k}\left(\gamma_{k}\right) \mathrm{d} \gamma .
$$

\section{Assumptions on the distribution function $\phi_{k}($.}

Since $\phi_{k}($.$) represents the demand function, we assume that \forall \gamma>0, \phi_{k}(\gamma)>0$. It 
is reasonable to assume that the more $\gamma$ (the unit price valuation) increases, the smaller the demand becomes, hence that $\phi_{k}(\gamma) \rightarrow 0$ when $\gamma \rightarrow \infty$.

For $q \in] 0, \bar{q}_{k}$ ], let's consider the following equation with variable $\alpha$ :

$$
q=\int_{\gamma \in \Gamma_{k}\left(T_{k}^{c}+\alpha \times a_{k}\right)} \phi_{k}(\gamma) \mathrm{d} \gamma
$$

Under the assumptions on $\phi_{k}($.$) , using the well known theorem on implicit func-$ tions, one can easily show that this equation has a unique solution $\alpha$; we will denote it $\alpha_{k}(q)$ and it is simply given by:

- if $a_{k}>0$ :

$$
\left.\left.q=\int_{\alpha_{k}(q)}^{+\infty} \phi_{k}(\gamma) \mathrm{d} \gamma \quad \forall q \in\right] 0, \bar{q}_{k}\right]
$$

- if $a_{k}<0$ :

$$
q=\int_{0}^{\alpha_{k}(q)} \phi_{k}(\gamma) \mathrm{d} \gamma \quad \forall q \in\left[0, \bar{q}_{k}\right]
$$

Thus, the function $\alpha_{k}($.$) is well defined on the interval \left[0, \bar{q}_{k}\right]$ if $a_{k}<0$ and on ] $\left.0, \bar{q}_{k}\right]$ if $a_{k}>0$, with $\alpha_{k}(q) \rightarrow \infty$ when $q \rightarrow 0$.

Proposition 5.1. For $\left.q \in] 0, \bar{q}_{k}\right]$, the revenue function value $R_{k}(q)$ is given as follows

(1) if $\alpha_{k}(q)>\bar{\alpha}_{k}$ :

$$
R_{k}(q)=\left(T_{k}^{c}+\bar{\alpha}_{k} a_{k}\right) \times q
$$

(2) if $\alpha_{k}(q) \leq \bar{\alpha}_{k}$ :

$$
\begin{array}{rc}
R_{k}(q)=\max _{0 \leq \alpha \leq \bar{\alpha}_{k}} & \left(T_{k}^{c}+\alpha a_{k}\right) \int_{\gamma \in \Gamma_{k}\left(T_{k}^{c}+\alpha \times a_{k}\right)} \phi_{k}(\gamma) \mathrm{d} \gamma \\
\text { s.t. } & \alpha \in \Gamma_{k}\left(T_{k}^{c}+\alpha_{k}(q) \times a_{k}\right) .
\end{array}
$$

Proof. The case $\alpha_{k}(q)>\bar{\alpha}_{k}$ may happen only when $a_{k}>0$. In this case, $\forall \alpha \in$ $\left[0, \bar{\alpha}_{k}\right]$ we necessarily have $q<\int_{\gamma \in \Gamma_{k}\left(T_{k}^{c}+\alpha \times a_{k}\right)} \phi_{k}(\gamma) d \gamma$ and by Lemma 5.3:

$$
R_{k}\left(q, T_{k}^{c}+\alpha \times a_{k}\right)=\left(T_{k}^{c}+\alpha \times a_{k}\right) q .
$$

Therefore, in this case, $R_{k}(q)=\max _{0 \leq \alpha \leq \bar{\alpha}_{k}}\left(T_{k}^{c}+\alpha a_{k}\right) \times q=\left(T_{k}^{c}+\bar{\alpha}_{k} a_{k}\right) \times q$.

From Lemma 5.3 and the definition of $\alpha(q)$, we have, $\forall \alpha \notin \Gamma_{k}\left(T_{k}^{c}+\alpha_{k}(q) \times a_{k}\right)$ :

$$
R_{k}\left(q, T_{k}^{c}+\alpha \times a_{k}\right)=\left(T_{k}^{c}+\alpha \times a_{k}\right) q \leq\left(T_{k}^{c}+\alpha_{k}(q) \times a_{k}\right) q=R_{k}\left(q, T_{k}^{c}+\alpha_{k}(q) \times a_{k}\right) .
$$

Therefore, we can deduce that if $\alpha(q) \leq \bar{\alpha}$ :

$$
\begin{array}{r}
R_{k}(q)=\max _{0 \leq \alpha \leq \bar{\alpha}_{k}} \quad R_{k}\left(q, T_{k}^{c}+\alpha \times a_{k}\right) \\
\text { s.t. } \quad \alpha \in \Gamma_{k}\left(T_{k}^{c}+\alpha_{k}(q) \times a_{k}\right)
\end{array}
$$


and the result is deduced from Lemma 5.3.

Remark 5.2. If $a_{k}>0$, the revenue function remains linear with slope $T_{k}^{c}+\bar{\alpha}_{k} a_{k}$ on the interval $\left[0, \int_{\bar{\alpha}_{k}}^{+\infty} \phi_{k}(\gamma) \mathrm{d} \gamma\right]$.

Lemma 5.4. The revenue function value $R_{k}(q)$ remains constant on the interval $\left[\bar{q}_{k},+\infty[\right.$.

Proof. Indeed, $\forall q \geq \bar{q}_{k}$, we have $q \geq \int_{\gamma \in \Gamma_{k}(T)} \phi_{k}(\gamma) \mathrm{d} \gamma \forall T \geq 0$ and using Lemma 5.3 we obtain $R_{k}(q, T)=T \int_{\gamma \in \Gamma_{k}(T)} \phi_{k}(\gamma) \mathrm{d} \gamma$. Hence, $R_{k}(q, T)$ and then $R_{k}(q)$ remains constant on $\left[\bar{q}_{k},+\infty[\right.$.

Remark 5.3. It is also easy to prove that $R_{k}(q)$ is a non-decreasing function on the interval $\left[0, \bar{q}_{k}\right]$.

For $q \in\left[0, \bar{q}_{k}\right]$ such that $\alpha_{k}(q) \leq \bar{\alpha}_{k}$, let's introduce the following differentiable function:

$$
\theta_{k}(q)=\left(T_{k}^{c}+\alpha_{k}(q) a_{k}\right) q
$$

Recall that the set of $q$ such that $q \in\left[0, \bar{q}_{k}\right]$ and $\alpha_{k}(q) \leq \bar{\alpha}_{k}$ is just the interval $\left[\underline{q}_{k}, \bar{q}_{k}\right]$ where $\underline{q}_{k}=\int_{\bar{\alpha}_{k}}^{+\infty} \phi_{k}(\gamma) d \gamma$ if $a_{k}>0$ and $\underline{q}_{k}=0$ if $a_{k}<0$.

Lemma 5.5. The function $\theta_{k}(q)$ is a lower bound function for the revenue function $R_{k}(q)$ :

$$
\theta_{k}(q) \leq R_{k}(q) \quad \forall q \in\left[\underline{q}_{k}, \bar{q}_{k}\right] .
$$

Proof. This result can be simply obtained by observing that $\theta_{k}(q)=R_{k}\left(q, T_{k}^{c}+\right.$ $\left.\alpha_{k}(q) \times a_{k}\right)$.

Lemma 5.6. The two following equalities are valid:

$$
\begin{gathered}
\forall q \in\left[\underline{q}_{k}, \bar{q}_{k}\right], R_{k}(q)=\max _{\underline{q}_{k} \leq q^{\prime} \leq q} \theta_{k}\left(q^{\prime}\right) \\
\max _{0 \leq q \leq \infty} R_{k}(q)=\max _{\underline{q}_{k} \leq q \leq \bar{q}_{k}} \theta_{k}(q) .
\end{gathered}
$$

Proof. From Proposition 5.1, we obtain

$$
\begin{aligned}
\forall q \in\left[\underline{q}_{k}, \bar{q}_{k}\right], R_{k}(q)=\max _{0 \leq q^{\prime}}\left(T_{k}^{c}+\alpha_{k}\left(q^{\prime}\right) a_{k}\right) q^{\prime} \\
\text { s.t. } \quad \alpha_{k}\left(q^{\prime}\right) \in \Gamma_{k}\left(T_{k}^{c}+\alpha_{k}(q) \times a_{k}\right) .
\end{aligned}
$$

According to the definition of $\theta_{k}(q)$ and using the fact that $\alpha_{k}\left(q^{\prime}\right) \in \Gamma_{k}\left(T_{k}^{c}+\right.$ $\left.\alpha_{k}(q) \times a_{k}\right)$ is equivalent to $q^{\prime} \leq q$, we obtain $R_{k}(q)=\max _{q \leq q^{\prime} \leq q} \theta_{k}\left(q^{\prime}\right)$.

The second equality is an immediate consequence of the first one.

In the sequel, we will study the concavity properties of the revenue function $R_{k}(q)$. 


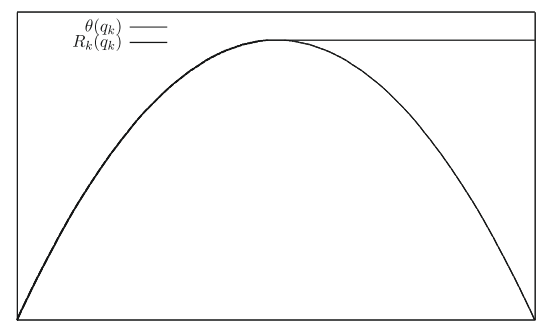

Figure 2. $\theta_{k}(q)$ and $R_{k}(q)$ concave.

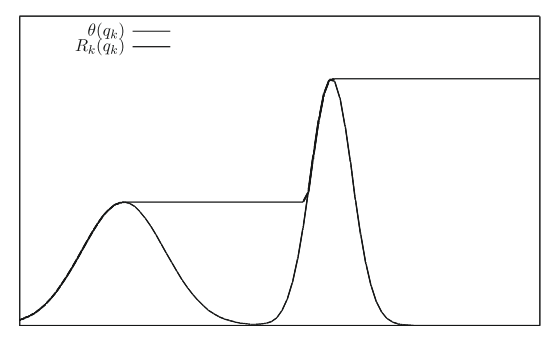

FiguRE $3 . \theta_{k}(q)$ and $R_{k}(q)$ non-concave.

Proposition 5.2. If the function $\theta_{k}(q)$ is concave on $\left[\underline{q}_{k}, \bar{q}_{k}\right]$ then the revenue function $R_{k}(q)$ is also concave on $[0,+\infty[$.

Proof. Using the previous lemma, $R_{k}(q)$ is the optimal value function of a concave maximization problem and then $R_{k}(q)$ is necessarily concave on $\left[\underline{q}_{k}, \bar{q}_{k}\right]$. If $\underline{q}_{k}>0$ we have shown (Rem. 5.2) that $R_{k}(q)$ is linear on $\left[0, \underline{q}_{k}\right]$ with slope $T_{k}^{c}+\bar{\alpha}_{k} a_{k}$. In this case we also have:

and

$$
R_{k}\left(\underline{q}_{k}\right)=\theta_{k}\left(\underline{q}_{k}\right)
$$

Thus, $R_{k}(q)$ is concave on $[0,+\infty[$.

$$
\theta_{k}^{\prime}(q)<T_{k}^{c}+\bar{\alpha}_{k} a_{k} \forall q>\underline{q}_{k}
$$

Remark 5.4. Assume here that $\theta_{k}($.$) is concave and let's note \hat{q}_{k}$ its maximum on $\left[\underline{q}_{k}, \bar{q}_{k}\right]$.

- We necessarily have $R_{k}(q)=\theta_{k}(q)$ if $\underline{q}_{k} \leq q \leq \hat{q}_{k}$ and $R_{k}(q)=\theta_{k}\left(\hat{q}_{k}\right)$ if $q>\hat{q}_{k}$ (see Figs. 2, 3).

- If $\theta_{k}^{\prime}\left(\bar{q}_{k}\right) \leq 0$ then $R_{k}(q)$ is necessarily differentiable on $] \underline{q}_{k},+\infty[$. Indeed, we have $\hat{q}_{k} \leq \bar{q}_{k}$ and $\theta_{k}^{\prime}\left(\hat{q}_{k}\right)=0$.

- If $\theta_{k}^{\prime}\left(\bar{q}_{k}\right)>0$ then $\hat{q}_{k}=\bar{q}_{k}$ and $R_{k}(q)$ remains non-differentiable on $\hat{q}_{k}$.

- If $a_{k}>0, R_{k}(q)$ also remains non-differentiable on $\underline{q}_{k}$.

Now, we will analyze conditions under which the function $\theta_{k}($.$) is concave.$ 


\section{Lemma 5.7.}

1. If the distribution function $\phi_{k}(\alpha)$ is $\mathcal{C}^{n}$ (i.e., n-times differentiable) then the function $\theta_{k}($.$) is \mathcal{C}^{n+1}$.

2. The derivative of the function $\alpha_{k}(q)$ is given by:

$$
\frac{\partial \alpha_{k}(q)}{\partial q}=\frac{-\epsilon_{k}}{\phi_{k}\left(\alpha_{k}(q)\right)}
$$

where $\epsilon_{k}=1$ if $a_{k}>0$ and $\epsilon_{k}=-1$ otherwise.

3. The function $\alpha_{k}(q)$ is decreasing if $a_{k}>0$ and increasing otherwise.

4. If $\phi_{k}(q)$ is differentiable then the second derivative of $\theta_{k}(q)$ is given by:

$$
\theta_{k}^{\prime \prime}(q)=-\epsilon_{k} \frac{a_{k}}{\phi_{k}\left(\alpha_{k}(q)\right)}\left(2+\epsilon_{k} q \frac{\phi_{k}^{\prime}\left(\alpha_{k}(q)\right)}{\left(\phi_{k}\left(\alpha_{k}(q)\right)\right)^{2}}\right) .
$$

Proof. If $\phi_{k}(\alpha)$ is $\mathcal{C}^{n}$, the function $\alpha_{k}(q)$ will be $\mathcal{C}^{n+1}$ and hence, $\theta_{k}($.$) is also \mathcal{C}^{n+1}$. The remaining results can be simply deduced by computing the first and second derivatives of the equation

$$
q=\int_{\gamma \in \Gamma_{k}\left(T_{k}^{c}+\alpha(q) \times a_{k}\right)} \phi_{k}(\gamma) \mathrm{d} \gamma
$$

to obtain:

$$
\begin{gathered}
\frac{\partial \alpha_{k}(q)}{\partial q} \phi_{k}\left(\alpha_{k}(q)\right)=-\epsilon_{k} \\
\phi_{k}^{\prime}\left(\alpha_{k}(q)\right)\left(\frac{\partial \alpha_{k}(q)}{\partial q}\right)^{2}+\phi_{k}\left(\alpha_{k}(q)\right) \frac{\partial^{2} \alpha_{k}(q)}{\partial^{2} q}=0
\end{gathered}
$$

and using that:

$$
\begin{aligned}
\theta_{k}^{\prime}(q) & =\left(T_{k}^{c}+\alpha_{k}(q) a_{k}\right)+q \frac{\partial \alpha_{k}(q)}{\partial q} a_{k} \\
\theta_{k}^{\prime \prime}(q) & =2 a_{k} \frac{\partial \alpha_{k}(q)}{\partial q}+q \frac{\partial^{2} \alpha_{k}(q)}{\partial^{2} q} a_{k} .
\end{aligned}
$$

Proposition 5.3. The function $\theta_{k}(q)$ remains concave over each interval $\left[q_{1}, q_{2}\right]$ such that, on the interval $\alpha_{k}\left(\left[q_{1}, q_{2}\right]\right)$, the distribution function is increasing (respectively decreasing) if $a_{k}>0$ (respectively $a_{k}<0$ )

Proof. From the previous lemma, one can easily see that $\theta_{k}^{\prime \prime}(q) \leq 0 \forall q \in\left[q_{1}, q_{2}\right]$

Now let:

$$
\begin{gathered}
q_{k}(\alpha)=\int_{\gamma \in \Gamma_{k}\left(T_{k}^{c}+\alpha \times a_{k}\right)} \phi_{k}(\gamma) \mathrm{d} \gamma \\
\Delta_{1}^{k}(\alpha)=\frac{\phi_{k}^{\prime}(\alpha)}{\phi_{k}(\alpha)} \\
\Delta_{2}^{k}(\alpha)=\frac{q_{k}^{\prime}(\alpha)}{q_{k}(\alpha)} .
\end{gathered}
$$


The quantities $\Delta_{1}^{k}(\alpha)$ and $\Delta_{2}^{k}(\alpha)$ respectively represent the relative variations of $\phi_{k}(\alpha)$ and $q_{k}(\alpha)$. Note that in fact $q_{k}(\alpha)$ is independent of data $T_{k}^{c}$ and $a_{k}$ since $\Gamma_{k}\left(T_{k}^{c}+\alpha \times a_{k}\right)$ is either $[\alpha,+\infty[$ or $[0, \alpha]$.

Theorem 5.1. If the distribution function $\phi_{k}(\alpha)$ is differentiable, then the function $\theta_{k}($.$) is concave if and only if:$

$$
\frac{\Delta_{1}^{k}(\alpha)}{\Delta_{2}^{k}(\alpha)} \leq 2 \quad \forall \alpha \in\left[0, \bar{\alpha}_{k}\right]
$$

Proof. Being twice differentiable, the function $\theta_{k}($.$) is concave if and only if \theta_{k}^{\prime \prime}(q) \leq$ $0 \forall q \geq 0$. The result is then directly obtained using the previous lemma, remarking that $q_{k}^{\prime}(\alpha)=-\epsilon_{k} \phi_{k}(\alpha)$ and using definitions of $\Delta_{1}^{k}(\alpha)$ and $\Delta_{2}^{k}(\alpha)$.

Using this result and Proposition 5.2 we obtain:

Corollary 5.1. The function $R_{k}($.$) is concave if \frac{\Delta_{1}^{k}(\alpha)}{\Delta_{2}^{k}(\alpha)} \leq 2 \quad \forall \alpha \in\left[0, \bar{\alpha}_{k}\right]$.

Remark 5.5 (elasticity condition). The ratio $\frac{\Delta_{1}^{k}(\alpha)}{\Delta_{2}^{k}(\alpha)}$ may be interpreted as what is commonly called the elasticity coefficient of the quantity $q_{k}(\alpha)$ related to $\phi_{k}(\alpha)$. The concavity property of the revenue function is then ensured when this elasticity is less than 2. Recall that $\phi_{k}(\alpha)$ represents the demand of the clients that could pay at most $T_{k}^{c}+\alpha a_{k}$. The quantity $q_{k}(\alpha)$ is the total demand of the clients that could pay at least $T_{k}^{c}+\alpha a_{k}$.

Remark 5.6. In economic theory, demand distribution functions are generally supposed to be concave, or less restrictively log-concave [3]. One can observe that if $q_{k}(\alpha)$ is supposed log-concave (i.e., $\log q_{k}(\alpha)$ is concave), then $\frac{\Delta_{1}^{k}(\alpha)}{\Delta_{2}^{k}(\alpha)} \leq 1$ and the necessary and sufficient condition (8) on the concavity of $\theta_{k}($.$) is satisfied. This$ necessary and sufficient condition is thus less restrictive than log-concavity.

Bergstrom and Bagnoli [3] show that if a probability density function (pdf) is log-concave, then its cumulative distribution function (cdf) is also log-concave. In our model, $q_{k}(\alpha)$ can be defined as the cdf of the pdf $\phi_{k}(\alpha)$; thus, if $\phi_{k}(\alpha)$ is $\log$-concave, then $q_{k}(\alpha)$ is also $\log$-concave and the function $\theta_{k}($.$) is concave.$

Remark 5.7. It is very interesting to observe that this ratio and then the concavity condition remain completely independent of the values of data parameters $T^{c}$ and $a_{k}$. This advantage comes from the fact that we work with the capacity allocations as decision variables. Indeed, instead of optimizing function $\theta_{k}(q)$, for instance, using $\alpha$ as a decision variable, one could work with the equivalent function $\psi_{k}(\alpha)$ given by

$$
\psi_{k}(\alpha)=\left(T_{k}^{c}+\alpha a_{k}\right) \int_{\alpha}^{\infty} \phi_{k}(\gamma) \mathrm{d} \gamma .
$$

It is easy to show that, contrarily to $\theta_{k}(q)$, the concavity condition of $\psi_{k}(\alpha)$ remains data $\left(T_{k}^{c}, a_{k}\right)$ dependent: for instance, it may not hold when $T_{k}^{c}$ is large. 


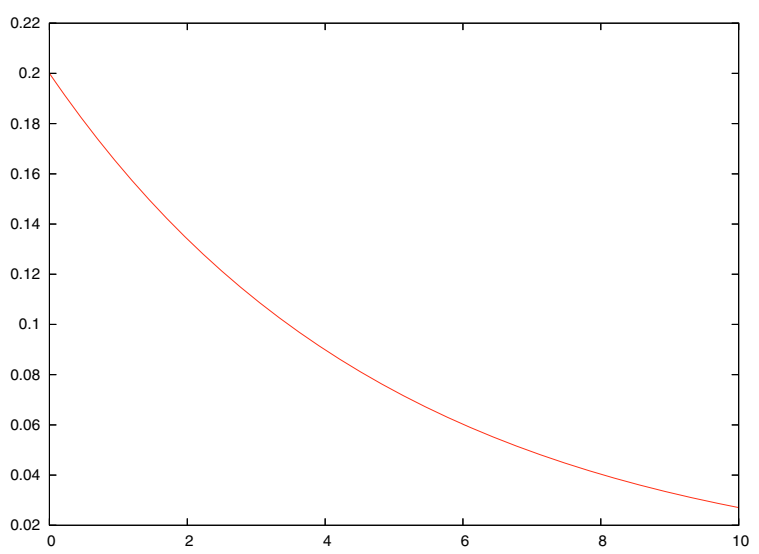

Figure 4 . Weibull $\beta=1, \eta=5$.

\section{WeIBUlL Distribution FUnCTION CASE STUDY}

The Weibull distribution is a widely used lifetime distribution. Its historical use is in engineering, where it is used to forecast machine failures over time. It has also been used in climatology (wind speed statistical modeling [16]), finance (option pricing [26]), or economics (reservation price distribution [1]). This distribution has two main advantages: it is easy to work with analytically (its integral can be written in closed form), and it can fit different distributions with adequately chosen parameters and thus is very versatile.

The probability density function of the Weibull distribution is:

$$
W(\alpha, \beta, \eta)=\frac{\beta}{\eta} \times\left(\frac{\alpha}{\eta}\right)^{(\beta-1)} \times \mathrm{e}^{-\left(\frac{\alpha}{\eta}\right)^{\beta}}
$$

where $\beta>0$ and $\eta>0$ are respectively the shape and the scale parameter. Figures 4 to 7 show how the curve of the function $W(\alpha, \beta, \eta)$ looks like when one increases the shape parameter $\beta$ starting from 1 (the parameter $\eta$ is kept constant).

Before giving the main result of this section, let's state the following lemma:

Lemma 6.1. Let $0 \leq t<1$. Then

$$
\mathrm{e}^{t} \leq \frac{1+t}{1-t}
$$

Proof. Let's consider the function $f(t)=(1-t) \mathrm{e}^{t}-(1+t)$. We have $f(0)=0$ and $f(1)=-2$. The derivative of this function is $-1-t \mathrm{e}^{t}<0$, i.e., the function is decreasing between 0 and -2 . Hence, $f(t) \leq 0$ for $0 \leq t \leq 1$ and the result follows. 


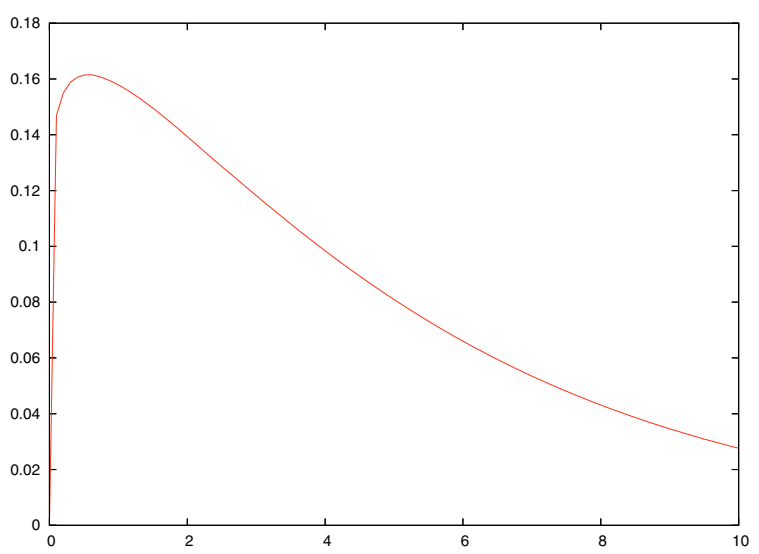

Figure 5. Weibull $\beta=1.1, \eta=5$.

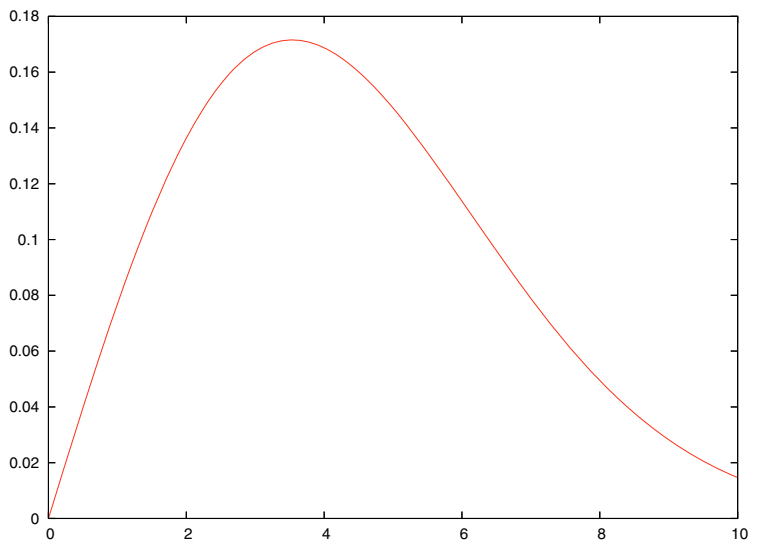

Figure 6 . Weibull $\beta=2, \eta=5$.

Theorem 6.1. If the demand distribution functions $\phi_{k}(\alpha)$ are of Weibull type, i.e., $\phi_{k}(\alpha)=W\left(\alpha, \beta_{k}, \eta_{k}\right)$ where $\beta_{k} \geq 1$ and $\eta_{k} \geq 0$, then the resource allocation optimization problem $(\tilde{P})$ (defined in Sect. 5 ) is concave.

Proof. The proof of the result is based on Corollary 5.1. Thus, we will calculate the quantities $\Delta_{1}^{k}(\alpha)$ and $\Delta_{2}^{k}(\alpha)$. To simplify, we will miss out the indices $k$ in the parameters $\beta_{k}, \eta_{k}$. Exploiting exponential function properties, one can obtain:

$$
\phi_{k}^{\prime}(\alpha)=\phi_{k}(\alpha)\left(\frac{\beta-1}{\alpha}-\frac{\beta}{\eta}\left(\frac{\alpha}{\eta}\right)^{\beta-1}\right)
$$




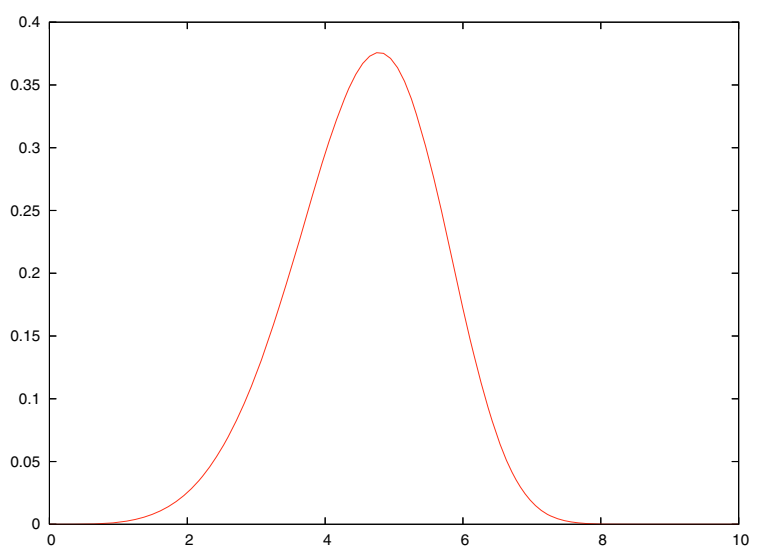

Figure 7. Weibull $\beta=5, \eta=5$.

and then:

$$
\Delta_{1}^{k}(\alpha)=\frac{\phi_{k}^{\prime}(\alpha)}{\phi_{k}(\alpha)}=\frac{\beta-1}{\alpha}-\frac{\beta}{\eta}\left(\frac{\alpha}{\eta}\right)^{\beta-1} .
$$

We also have

$$
\int_{\alpha}^{+\infty} \phi_{k}(\alpha) \mathrm{d} \alpha=\mathrm{e}^{-\left(\frac{\alpha}{\eta}\right)^{\beta}}
$$

and

$$
\int_{0}^{\alpha} \phi_{k}(\gamma) \mathrm{d} \gamma=1-\mathrm{e}^{-\left(\frac{\alpha}{\eta}\right)^{\beta}}
$$

Therefore:

- if $a_{k}>0$ :

$$
q_{k}(\alpha)=\mathrm{e}^{-\left(\frac{\alpha}{\eta}\right)^{\beta}} \quad q_{k}^{\prime}(\alpha)=-\phi_{k}(\alpha)
$$

and

$$
\Delta_{2}^{k}(\alpha)=\frac{q_{k}^{\prime}(\alpha)}{q_{k}(\alpha)}=-\frac{\beta}{\eta} \times\left(\frac{\alpha}{\eta}\right)^{(\beta-1)} .
$$

In this case, we obtain:

$$
\Delta_{1}^{k}(\alpha)-2 \Delta_{2}^{k}(\alpha)=\frac{\beta-1}{\alpha}+\frac{\beta}{\eta}\left(\frac{\alpha}{\eta}\right)^{\beta-1} \geq 0 \quad \forall \alpha \geq 0 .
$$

Since $\Delta_{2}^{k}(\alpha) \leq 0$ we deduce that

$$
\frac{\Delta_{1}^{k}(\alpha)}{\Delta_{2}^{k}(\alpha)} \leq 2
$$


- if $a_{k}<0$

$$
q_{k}(\alpha)=1-\mathrm{e}^{-\left(\frac{\alpha}{\eta}\right)^{\beta}} \quad q_{k}^{\prime}(\alpha)=\phi_{k}(\alpha)
$$

and

$$
\Delta_{2}^{k}(\alpha)=\frac{q_{k}^{\prime}(\alpha)}{q_{k}(\alpha)}=\frac{\frac{\beta}{\eta} \times\left(\frac{\alpha}{\eta}\right)^{(\beta-1)} \times \mathrm{e}^{-\left(\frac{\alpha}{\eta}\right)^{\beta}}}{1-\mathrm{e}^{-\left(\frac{\alpha}{\eta}\right)^{\beta}}} .
$$

Let $t=\left(\frac{\alpha}{\eta}\right)^{\beta}$ and $u=\frac{(\beta-1)}{\beta} \times \eta$.

In this case, one can easily deduce that:

$$
\begin{aligned}
\frac{\eta}{\beta} \times \alpha \times\left(e^{t}-1\right) \times\left(\Delta_{1}^{k}(\alpha)-2 \Delta_{2}^{k}(\alpha)\right) & =e^{t}(u-\eta t)-(u+\eta t) \\
& =u\left(e^{t}(1-\omega)-(1+\omega)\right) .
\end{aligned}
$$

where $\omega=\frac{\eta t}{u}$.

If $\omega \geq 1$, we directly obtain that $\Delta_{1}^{k}(\alpha)-2 \Delta_{2}^{k}(\alpha) \leq 0$.

Otherwise, if $\omega<1$, using the previous lemma and remarking that $t \leq \omega$, we obtain:

$$
\mathrm{e}^{t} \leq \mathrm{e}^{\omega} \leq \frac{1+\omega}{1-\omega}
$$

Thus, we also obtain

$$
\Delta_{1}^{k}(\alpha)-2 \Delta_{2}^{k}(\alpha) \leq 0 .
$$

Since $\Delta_{2}^{k}(\alpha) \leq 0$, finally, we deduce that

$$
\frac{\Delta_{1}^{k}(\alpha)}{\Delta_{2}^{k}(\alpha)} \leq 2 .
$$

As an example, Figure 8 illustrates the concavity of $\theta_{k}(q)$, and thus $R_{k}(q)$, w.r.t. the capacity variable $q$. For this example, in Figure 9 the revenue function $\psi_{k}(\alpha)$ w.r.t. the variable $\alpha$, defined in Remark 5.7, is drawn. One can observe that, unlike $R_{k}(q)$, the revenue function represented by $\psi_{k}(\alpha)$ is not concave everywhere. Indeed, in Figure $9 \psi_{k}(\alpha)$ is nearly convex in the second part of the curve. As mentioned before, this shows that it will be more convenient to work with $R_{k}(q)$ rather than $\psi_{k}(\alpha)$ since the resource allocation optimization problem remains concave and might be efficiently solved with classical convex nonlinear mathematical programming algorithms.

\section{Solution methods}

Based on the results obtained in the previous sections and in previous work [7], we investigate two solution methods to solve the joint pricing and resource allocation problem. 


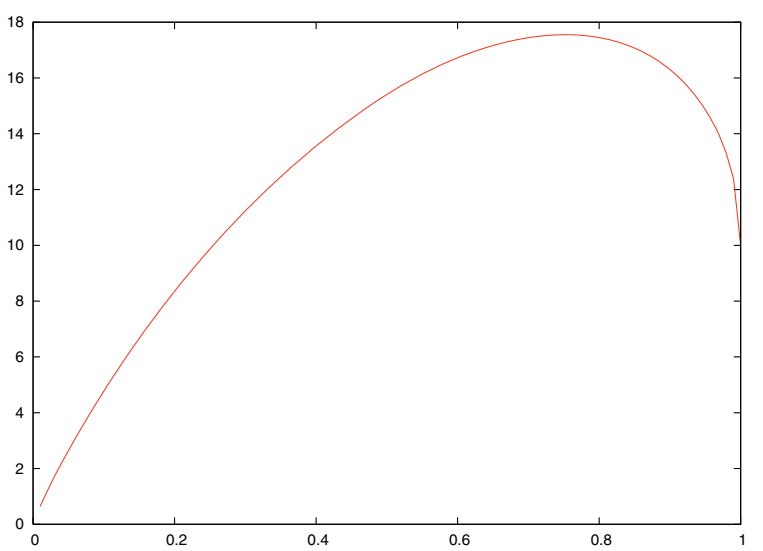

Figure 8. $\theta_{k}(q)(\beta=2, \eta=5)$.

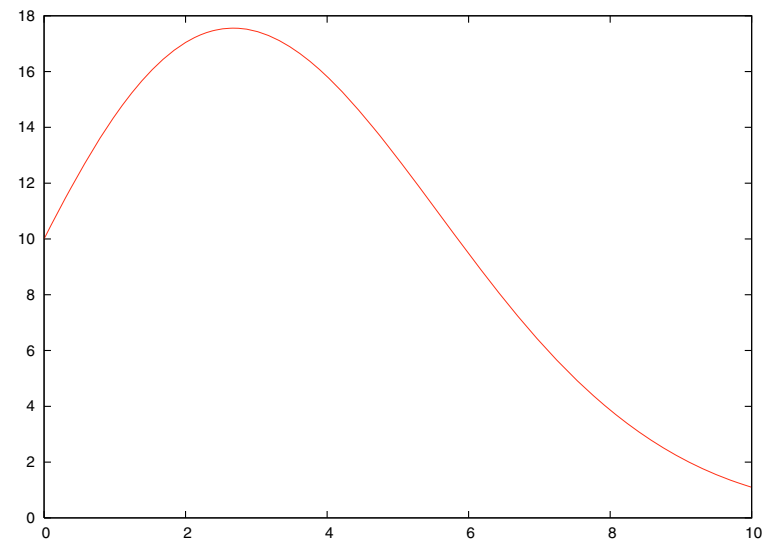

Figure 9. $\psi_{k}(\alpha)(\beta=2, \eta=5)$.

On the one hand, the problem $\left(P_{c}\right)$ can be handled directly using nonlinear continuous optimization techniques and solvers. On the other hand, for marketing practitioners, it might be more natural to consider discrete market segments; in this case, a good discretization of the continuous distribution function must be found so that the model can be well approximated. The resulting model can then be solved using the methods described in Section 4.2 and [7].

In the next sections, we introduce methodologies to solve the problem, first in the nonlinear continuous context, then using the discretization approach. We finally give numerical results on telecommunications networks instances. 


\subsection{NONLINEAR CONTINUOUS OPTIMIZATION APPROACH}

According to the results obtained in the previous sections, the joint pricing and resource allocation optimization problem $\left(P_{c}\right)$ can be solved in two steps:

(1) Computing capacities to allocate to each market by solving

$$
\begin{aligned}
& \max _{q_{k}} \sum_{k \in \mathcal{K}} R_{k}\left(q_{k}\right) \\
& \text { (I) } \quad \text { s.t. } \quad \sum_{k \in \mathcal{K}} \sum_{k \mid a \in \mathcal{A}(k)} q_{k} \leq c_{a} \quad \forall a \in \mathcal{A} \\
& q_{k} \geq 0 \text {. }
\end{aligned}
$$

(2) Recovering the optimal tariffs associated with these optimal capacities.

Functions $R_{k}($.$) are non-smooth and non-concave in the general case. We have$ shown in Proposition 5.2 that when $\theta_{k}($.$) is concave on \left[\underline{q}_{k}, \bar{q}_{k}\right], R_{k}($.$) is also$ concave on $[0,+\infty[$ and its curve is formed with linear and nonlinear concave pieces (see Fig. 2).

More precisely, in this case, the objective function to consider is as follows:

$$
R_{k}\left(q_{k}\right)= \begin{cases}\bar{T}_{k} \cdot q_{k} & 0 \leq q_{k} \leq \underline{q}_{k} \\ \theta_{k}\left(q_{k}\right) & \underline{q}_{k} \leq q_{k} \leq \hat{q}_{k} \\ \theta_{k}\left(\hat{q}_{k}\right) & \hat{q}_{k} \leq q_{k} .\end{cases}
$$

We recall that $\bar{T}_{k}$ is the bound on the possible prices; $\underline{q}_{k}$ is the potential demand captured when the tariff is $\bar{T}_{k} ; \hat{q}_{k}$ is the capacity for which $\theta_{k}\left(q_{k}\right)$ is maximum on $\left[\underline{q}_{k}, \bar{q}_{k}\right]$. Of course, the latter must be computed, which can be easily done for example by a Newton-like method when $\theta_{k}($.$) is concave.$

Therefore, in this case, this typical problem $(\tilde{P})$ could be simply formulated as a concave and differentiable problem.

The nonlinear optimization problem that we obtain can thus be solved using classical nonlinear optimization methods. In Section 7.3, we present numerical results obtained with an interior point methods, using the IPOPT software package [27].

When $\theta_{k}($.$) is concave, we have R_{k}\left(q_{k}\right)=\left(T_{k}^{c}+\alpha_{k}\left(q_{k}\right) a_{k}\right) q_{k}$, and then the optimal tariffs can simply be recovered by setting $T_{k}=T_{k}^{c}+\alpha_{k}\left(q_{k}\right) a_{k}$.

Remark 7.1. If the demand distribution function $\phi_{k}($.$) is a Weibull distribution$ function, the first and second derivatives of the function $\theta_{k}($.$) can be easily com-$ puted, if needed by the solution algorithm, as is the case with an interior point method for example. To recover optimal tariffs, $\alpha_{k}\left(q_{k}\right)$ is also easy to compute in this case. 


\subsection{Demand FUnCtion Discretization AND MiXed INTEGER LiNEAR OPTIMIZATION APPROACH}

In order to solve the problem $\left(P_{c}\right)$ using the discrete approach presented in Section 4.2 , a discretization of the distribution function $\phi_{k}($.$) must be defined.$ The following questions must be answered: what interval should we sample?; which sample values should we choose?; which values of the demand should we assign to these values?

Let's recall that $\phi_{k}($.$) is defined in the unbounded interval [0,+\infty[$. However, we show that we can work only in a bounded interval.

Property 7.1. In the continuous optimization problem $\left(P_{c}\right)$, the function $\phi_{k}($. can be replaced with the following distribution function $\hat{\phi}_{k}($.$) which is only defined$ in the bounded interval $\left[0, \bar{\alpha}_{k}\right]$ :

$$
\begin{array}{lll}
\text { if } a_{k}<0 & \hat{\phi}_{k}(\gamma)=\phi_{k}(\gamma) & 0 \leq \gamma \leq \bar{\alpha}_{k} \\
\text { if } a_{k}>0 & \hat{\phi}_{k}(\gamma)=\phi_{k}(\gamma) & 0 \leq \gamma<\bar{\alpha}_{k} \\
& \hat{\phi}_{k}\left(\bar{\alpha}_{k}\right)=\int_{\bar{\alpha}_{k}}^{+\infty} \phi_{k}(\gamma) \mathrm{d} \gamma . &
\end{array}
$$

Proof. For $a_{k}<0$, every market segment $\gamma>\bar{\alpha}_{k}$ perceives the competition offer as cheaper (to obtain market segment $\gamma>\bar{\alpha}_{k}$, the operator should set the tariff of offer $k$ to $T_{k}^{c}+\gamma a_{k}<0$, but we impose the constraint $\left.T_{k} \geq 0\right)$. For $a_{k}>0$, every market segment $\gamma>\bar{\alpha}_{k}$ perceives the operator offer as cheaper $\left(\gamma>\bar{\alpha}_{k} \Rightarrow\right.$ $\left.T_{k}^{c}+\gamma D_{k}^{c}>T_{k}^{c}+\bar{\alpha}_{k} D_{k}^{c}=\bar{T}_{k}+\bar{\alpha}_{k} D_{k}\right)$. Thus, in this case, one can aggregate the demand of market segments $\gamma \geq \bar{\alpha}_{k}$ as the demand value to assign to the segment $\alpha_{k}$.

Hence, we will discretize the function whose value is $\phi_{k}(\gamma)$ for $\gamma \in\left[0, \bar{\alpha}_{k}\right]$ if $a_{k}<0, \phi_{k}(\gamma)$ for $\gamma \in\left[0, \bar{\alpha}_{k}\left[\right.\right.$ and $\int_{\bar{\alpha}_{k}}^{+\infty} \phi_{k}(\gamma) \mathrm{d} \gamma$ in $\bar{\alpha}_{k}$ if $a_{k}>0$.

We choose to take a uniform sampling of $\left[0, \bar{\alpha}_{k}\right]$ as $\left\{\gamma_{0}=0, \ldots, \gamma_{N}=\bar{\alpha}_{k}\right\}$ $\left(\gamma_{i}\right.$ 's should also be indexed by $k$; we omit these indices here for simplicity of the notation).

We choose to assign all the demand $\int_{\gamma_{i}}^{\gamma_{i}+1} \phi_{k}(\gamma) \mathrm{d} \gamma$ of segments between $\gamma_{i}$ and $\gamma_{i+1}$ to the segment $\gamma_{i}$ (respectively $\gamma_{i+1}$ ) when $a_{k}>0$ (respectively $a_{k}<0$ ). More specifically, we define the discrete distribution function $\tilde{\phi}($.$) as follows:$

$$
\begin{aligned}
& \text { if } a_{k}<0 \quad \tilde{\phi}_{k}\left(\gamma_{0}\right)=0 \\
& \tilde{\phi}_{k}\left(\gamma_{i}\right)=\int_{\gamma_{i-1}}^{\gamma_{i}} \phi_{k}(\gamma) \mathrm{d} \gamma \quad 0<i \leq N \text {; }
\end{aligned}
$$




$$
\begin{aligned}
& \text { if } a_{k}>0 \quad \tilde{\phi}_{k}\left(\gamma_{i}\right)=\int_{\gamma_{i}}^{\gamma_{i+1}} \phi_{k}(\gamma) \mathrm{d} \gamma \quad 0 \leq i<N \\
& \tilde{\phi}_{k}\left(\gamma_{N}\right)=\int_{\bar{\alpha}_{k}}^{+\infty} \phi_{k}(\gamma) \mathrm{d} \gamma .
\end{aligned}
$$

Remark 7.2. These choices are justified by the following remarks:

- If the value of the discretized function at each $\gamma_{i}$ is defined as $\phi_{k}\left(\gamma_{i}\right)$, not all the demand is taken into account and the discretized model is not equivalent to the continuous one.

- If the integral $\int_{\gamma_{i}}^{\gamma_{i}+1} \phi_{k}(\gamma) \mathrm{d} \gamma$ is rather assigned to some segment in the interval $] \gamma_{i}, \gamma_{i+1}\left[\left(\frac{\gamma_{i}+\gamma_{i+1}}{2}\right.\right.$ for example), the corresponding discrete model will not be equivalent to the continuous one. Indeed, the same tariff should not allow the operator to capture the same amount of demand in both models.

We now analyze the links between the continuous and the discrete model obtained using the discretized distribution function $\tilde{\phi}($.) defined above.

Property 7.2. In both models, the same potential demand $q_{k}\left(\gamma_{j}\right)$ could be captured by the operator when targeting the segment $\gamma_{j}$ by setting the tariff to $T_{k}^{c}+\gamma_{j} a_{k}$.

Proof. For $a_{k}>0$, when targeting the segment $\gamma_{j}$, every market segment $\gamma>\gamma_{j}$ perceives the operator offer as cheaper $\left(T_{k}^{c}+\gamma D_{k}^{c}>T_{k}^{c}+\gamma_{j} D_{k}^{c}\right)$. In this case, the potential demand the operator could be captured is simply given by $q_{k}\left(\gamma_{j}\right)=$ $\int_{\gamma_{j}}^{+\infty} \phi_{k}(\gamma) \mathrm{d} \gamma$ in the continuous model and by $q_{k}^{\text {discrete }}\left(\gamma_{j}\right)=\sum_{\gamma_{j} \leq \gamma_{i}} \tilde{\phi}_{k}\left(\gamma_{i}\right)$ in the discrete one.

Hence, by definition, we obtain:

$$
q_{k}^{\text {discrete }}\left(\gamma_{j}\right)=\sum_{\gamma_{j} \leq \gamma_{i}} \int_{\gamma_{i}}^{\gamma_{i+1}} \phi_{k}(\gamma) \mathrm{d} \gamma=q_{k}\left(\gamma_{j}\right)
$$

The same reasoning holds for $a_{k}<0$.

Now, as in the Section 7.1, we assume that the function $\theta_{k}($.$) defined in the$ continuous model is concave in the interval $\left[\underline{q}_{k}, \bar{q}_{k}\right]$ and we denote $\hat{q}_{k}$ its maximum.

Let $R_{k}^{\text {discrete }}(q)$ be the revenue function in the discrete model with respect to the capacity variable $q$. We have the following results:

Property 7.3. Both models give the same revenue value $R_{k}\left(q_{k}\left(\gamma_{j}\right)\right)$ when targeting a segment $\gamma_{j}$ such that $q_{k}\left(\gamma_{j}\right) \leq \hat{q}_{k}$ :

$$
R_{k}^{\text {discrete }}\left(q_{k}\left(\gamma_{j}\right)\right)=R_{k}\left(q_{k}\left(\gamma_{j}\right)\right) .
$$

Proof. We study the case $a_{k}>0$ (a similar reasoning holds for $a_{k}<0$ ). 


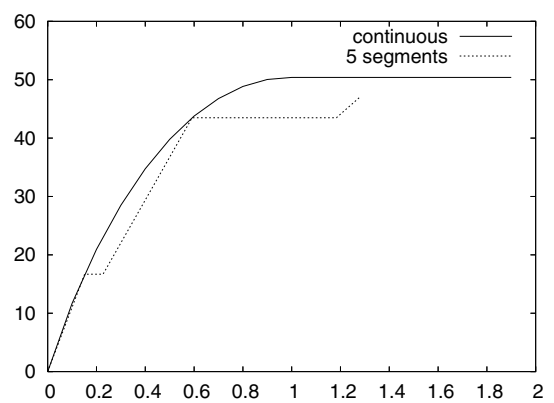

Figure 10. Objective functions for continuous model and discrete model with 5 market segments.

Recall that, as shown in Section 4.2 and in [7], the revenue function $R_{k}^{\text {discrete }}(q)$ of the discrete model is a non-decreasing piecewise linear function with alternating linear and constant pieces which are defined by

$$
R_{k}^{\text {discrete }}\left(q_{k}\left(\gamma_{j}\right)\right)=\max _{j \leq i}\left(\left(T_{k}^{c}+\gamma_{i} a_{k}\right) q_{k}\left(\gamma_{i}\right)\right)
$$

By definition of the continuous function $\theta_{k}($.$) , we have for all \gamma_{i}$ such that $\underline{q}_{k} \leq$ $q_{k}\left(\gamma_{i}\right) \leq \hat{q}_{k}$

$$
\theta_{k}\left(q_{k}\left(\gamma_{i}\right)\right)=\left(T_{k}^{c}+\gamma_{i} a_{k}\right) q_{k}\left(\gamma_{i}\right)
$$

Observing that $\underline{q}_{k}=q_{k}\left(\gamma_{N}\right)$ and $\forall i, \underline{q}_{k}\left(\gamma_{N}\right) \leq q_{k}\left(\gamma_{i+1}\right) \leq q_{k}\left(\gamma_{i}\right)$, we directly deduce that

$$
R_{k}^{\text {discrete }}\left(q_{k}\left(\gamma_{j}\right)\right)=\max _{i \geq j} \theta_{k}\left(q_{k}\left(\gamma_{i}\right)\right) \quad \forall j: q_{k}\left(\gamma_{j}\right) \leq \hat{q}_{k} .
$$

Concavity of the function $\theta_{k}($.$) implies that \theta_{k}($.$) is necessarily non-decreasing in$ $\left[\underline{q}_{k}, \hat{q}_{k}\right]$ and $\forall j: q_{k}\left(\gamma_{j}\right) \leq \hat{q}_{k}, R_{k}\left(q_{k}\left(\gamma_{j}\right)\right)=\theta_{k}\left(q_{k}\left(\gamma_{j}\right)\right)$. Thus, we obtain:

$$
R_{k}^{\text {discrete }}\left(q_{k}\left(\gamma_{j}\right)\right)=\theta_{k}\left(q_{k}\left(\gamma_{j}\right)\right)=R_{k}\left(q_{k}\left(\gamma_{j}\right)\right) \quad \forall j: q_{k}\left(\gamma_{j}\right) \leq \hat{q}_{k}
$$

and the result follows.

This property is illustrated in Figures 10 and 11. These figures also show that, using this discretization, the discrete problem that we have to solve approximates the corresponding continuous problem. The approximation gets better as the number of market segments (i.e., samplings) increases.

Property 7.4. The value of the revenue function $R_{k}(q)$ given by the continuous model is always over the one given by the discrete model.

Proof. By Property 7.3, for all $\gamma_{j}$ such that $q_{k}\left(\gamma_{j}\right) \leq \hat{q}_{k}$, we have $R_{k}^{\text {discrete }}\left(q_{k}\left(\gamma_{j}\right)\right)=$ $R_{k}\left(q_{k}\left(\gamma_{j}\right)\right)$. Now, let's consider the concave envelope of the function $R_{k}^{\text {discrete }}($.$) .$ 


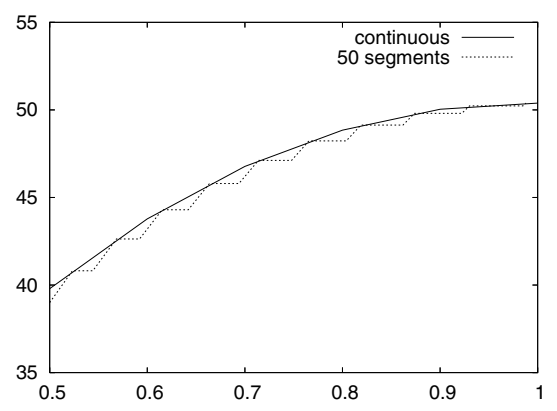

Figure 11. Objective functions for continuous model and discrete model with 50 market segments.

It is a piecewise linear function composed of the lines joining couples of points $\left(q_{k}\left(\gamma_{i}\right), R_{k}\left(q_{k}\left(\gamma_{i}\right)\right)\right.$. On the one hand, by construction, this concave envelope is over the function $R_{k}^{\text {discrete }}($.$) . On the other hand, this concave envelope is necessarily$ below the continuous concave function $R_{k}($.$) . Hence the result.$

Finally, in this discrete model, the corresponding optimization problem $(\tilde{P})$, defined in Section 4.2, can be formulated as a mixed integer linear program and solved for example using a branch-and-cut algorithm (see [7] for more details). However, as shown in [7], due to its combinatorial nature, this problem could be difficult to solve in practice.

Nevertheless, an approximate solution value to the MILP formulation can be satisfying. For example, the concave envelope of the functions $R_{k}^{\text {discrete }}($.$) can$ be used to compute a satisfying approximate solution. Moreover, one can observe that, this concave envelope approximates the continuous model better than $R_{k}^{\text {discrete }}($.$) . Also, note that this approximate solution is the solution of the linear$ programming relaxation of the mixed integer integer linear program [12].

\subsection{EXPERIMENTAL RESUlts}

In this section, we present some numerical results obtained on instances of the problem. The objective is to estimate the feasibility of solving the problem on realistic instances.

To solve the nonlinear continuous optimization model, we use the interior point method implemented by the IPOPT software package [27] (version of 2005/06/10). We consider a Weibull function for demand distribution on each market, i.e., the optimization problem has a concave objective, and linear constraints.

In Table 1, we give the results obtained. Three networks of different sizes are used, based on networks of France Telecom between French cities. The network topology and the routing are fixed, while arc capacities, offer qualities, demand and competition tariffs are randomly generated. For each network size, ten instances are generated this way. The table displays the minimum, the average and the 
TABLE 1. Numerical results using IPOPT on the nonlinear continuous model.

\begin{tabular}{c|c|rrr|rrr}
\hline $\mathcal{A} \mid$ & $|\mathcal{M}|$ & \multicolumn{3}{|c}{ Iterations } & \multicolumn{3}{c}{ CPU time } \\
& & $\min$ & avg & $\max$ & $\min$ & avg & $\max$ \\
\hline 16 & 28 & 28 & 33 & 38 & 0.44 & 0.49 & 0.57 \\
65 & 210 & 30 & 32 & 35 & 11.78 & 12.30 & 13.33 \\
105 & 319 & 27 & 29 & 32 & 22.11 & 23.90 & 25.28 \\
\hline
\end{tabular}

TABLE 2. CPU times and solution quality with 10 and 50 discretization steps, using CPLEX on the mixed integer linear model.

\begin{tabular}{r|r|r|rr|rr}
\hline $\mathcal{A} \mid$ & $|\mathcal{M}|$ & Instance & \multicolumn{2}{|c}{ Steps $=50$} & \multicolumn{2}{c}{ Steps $=10$} \\
& & & CPU time & Objective value & CPU time & Objective value \\
\hline 105 & 319 & 0 & 560 & 99.8468 & 531 & 98.6114 \\
& 1 & $24875^{*}$ & 99.8750 & 2372 & 98.7873 \\
& 2 & 3559 & 99.9032 & 75 & 98.7503 \\
& 3 & 15664 & 99.9341 & 272 & 99.3892 \\
& 4 & 3199 & 99.9022 & 50 & 99.0436 \\
& 5 & $19641^{*}$ & 99.8886 & 2750 & 98.9394 \\
& 6 & 20738 & 99.9077 & 723 & 98.9757 \\
& 7 & $12737^{*}$ & 99.8907 & 93 & 98.8393 \\
& 8 & 3216 & 99.8419 & 69 & 98.3283 \\
& 9 & 2310 & 99.8885 & 48 & 98.5798 \\
\hline
\end{tabular}

maximum number of iterations and CPU time taken by the interior point method on a $400 \mathrm{MHz}$ Sun UltraSparc II workstation.

As expected, the interior point method converges to an optimal solution and there is little variation of the number of iterations as the size of the instances increases. The optimal solution is obtained within a few seconds, even on the largest of the instances, which is quite satisfactory. The number of variables and constraints of the problem is small, which is an advantage of the continuous model.

As stated in Section 7.2, by discretizing the distribution function, the problem can be solved as a mixed integer linear program. This program was solved using the CPLEX software package (version 7.5) on the instances described before, using different discretization steps. In Table 2, we only retain the results (CPU time and solution quality in percentage of the continuous model solution value) obtained on the largest instances, with discretization steps 10 and 50.

These results show that the solution quality on these instances is very good, even with 10 discretization steps. However, this is at the cost of CPU time, which varies greatly from instance to instance. On some of the instances it can take a very long time to solve the problem, and sometimes even not to optimality before exhausting the $512 \mathrm{Mb}$ memory limit (these instances are denoted by '*').

Furthermore, the accuracy of the discretization depends on the value of $\bar{T}_{k}$ (that gives $\bar{\alpha}_{k}$ ); for some values, there can be an important gap between the solutions 
TABLE 3. CPU times and solution quality with 50 discretization steps, using CPLEX on the linear program for solving the concave relaxation of the problem.

\begin{tabular}{|c|c|c|c|c|c|c|c|}
\hline \multirow[t]{3}{*}{$\overline{|\mathcal{A}|}$} & \multirow[t]{3}{*}{$|\mathcal{M}|$} & \multicolumn{6}{|c|}{ Steps $=50$} \\
\hline & & \multicolumn{3}{|c|}{ CPU time } & \multicolumn{3}{|c|}{ Objective value } \\
\hline & & $\min$ & avg & $\max$ & $\min$ & avg & $\max$ \\
\hline 105 & 319 & 0.90 & 0.95 & 1.01 & 99.9092 & 99.9560 & 99.9872 \\
\hline
\end{tabular}

of the continuous and the discretized models. Indeed, if $\bar{\alpha}_{k}$ is far in the tail of the Weibull distribution, most of the discretization steps will be in the tail; thus, the price associated with most of the demand will be much lower than it would be with the continuous model. This is a practical limitation of this model.

As we showed in the previous section, it is not necessary to solve the MILP for the discretized problem in order to obtain an approximate solution of the continuous model. Indeed, the concave envelope of the objective function approximates the continuous function better and can be formulated as a linear program. In Table 3 , the minimum, the average and the maximum CPU time and solution quality for the largest instances are displayed, for 50 discretization steps.

We observe that the quality of the solution is very good, for very low CPU time. However, the comments on the value of $\bar{\alpha}_{k}$ apply again, and this can lead to bad solution qualities. Moreover, even if on the instances considered the quality of the solution is very good, this can only be confirmed by solving the continuous model. As the continuous model can be solved efficiently, we might as well solve it directly, unless tight solving time constraints are required.

\section{Conclusion}

This paper investigates a continuous optimization model for a revenue management problem in the context of the competitive telecommunications industry. This problem consists in simultaneously pricing point-to-point telecommunications services and allocating resources in the network, in the presence of competition. The market is segmented and each market segment has its own purchasing behavior.

This type of problems has classically been modeled as a bilevel program. We used another formulation for the problem, as introduced in [7]. In the case where the operator provides one offer on each market, we proved mathematical properties of the problem, and notably we gave a necessary and sufficient condition on the demand distribution function for convexity of the problem. We also gave numerical results on realistic instances of telecommunications networks.

Using a Weibull model for the demand distribution function, we solved the problem using an interior point method. As expected, the method converges to an optimal solution in a few iterations. Using the existing models for the discrete case, we analyzed the results obtained by discretizing the Weibull distribution function and solving a mixed integer linear program. On the largest instances, the 
branch-and-bound algorithm of the solver can fail to find an optimal solution in a reasonable time. The discrete model can also be approximated by taking the concave envelope of its objective function. In this case, we showed that the solution obtained is a good approximation of the solution of the continuous problem on the instances that were considered. However this approach can have practical limitations, depending on the values of the data of the problem, that the nonlinear model does not have.

In further work, we intend to investigate the properties of the problem in the case where the operator can provide several offers on each market. The above mathematical analysis cannot be conducted in the same way in this case. Indeed, in the present paper, the mathematical analysis of the problem is possible because an analytical definition of an optimal tariff can be given; however, if the operator provides several offers on each market, such an analytical definition is not easily obtained, as the tariff for each offer depends on the other tariffs.

Acknowledgements. We would like to thank Michel Minoux for his comments on an early version of this paper. We also would like to thank the anonymous referees for their insightful comments.

\section{REFERENCES}

[1] G. Aydin and J. Ryan, Product line selection with stochastic utility (2000) (submitted).

[2] M. Ben-Akiva and M. Bierlaire, Discrete choice methods and their applications to short term travel decisions, in Handbook of transportation science, edited by R. Hall, International Series in Operations Research and Management Science, Vol. 23. Kluwer Academic Publishers, Dordrecht (1999) 5-34.

[3] T. Bergstrom and M. Bagnoli, Log-concave probability and its applications. Econ. Theory 26 (2005) 445-469.

[4] M. Bouhtou, M. Diallo and L. Wynter, Capacitated network revenue management through shadow pricing, in Group communications and charges; technology and business models, edited by B. Stiller, G. Carle, M. Karsten and P. Reichl, Lect. Notes Comput. Sci. 2816, Springer-Verlag, Berlin, Heidelberg (2003) 342-352.

[5] M. Bouhtou, M. Diallo and L. Wynter, Fair network resource allocation and link pricing: A numerical study, in Optimization and optimal control, edited by P. Pardalos, I. Tsevendorj and R. Enkhbat, World Scientific Publishing, Singapore (2003) 37-58.

[6] M. Bouhtou, G. Erbs and M. Minoux, Pricing and resource allocation for point-to-point telecommunication services in a competitive market: a bilevel optimization approach, in Telecommunications planning: innovations in pricing, network design and management, edited by G. Anandalingam and S. Raghavan, Operations Research/Computer Science Interfaces Series, Vol. 33. Springer-Verlag, New York (2005) 1-16.

[7] M. Bouhtou, G. Erbs and M. Minoux, Joint optimization of pricing and resource allocation in competitive telecommunications networks. Networks 50 (2007) 37-49.

[8] M. Bouhtou, S. van Hoesel, A. van der Kraaij and J.-L. Lutton, Tariff optimization in networks. INFORMS J. Comput. 19 (2007) 458-469.

[9] L. Brotcorne, M. Labbé, P. Marcotte and G. Savard, A bilevel model and solution algorithm for a freight tariff setting problem. Transportation Science 34 (2000) 289-302.

[10] L. Brotcorne, M. Labbé, P. Marcotte, and G. Savard. A bilevel model for toll optimization on a multicommodity transportation network. Transportation Science 35 (2001) 1-14. 
[11] J.-P. Côté, P. Marcotte and G. Savard, A bilevel modelling approach to pricing and fare optimisation in the airline industry. J. Revenue Pricing Manag. 2 (2003) 23-36.

[12] K.L. Croxton, B. Gendron and T.L. Magnanti, A comparison of mixed-integer programming models for non-convex piecewise linear cost minimization problems. Manage. Sci. 49 (2003) $1268-1273$

[13] M. Fortin, P. Marcotte and G. Savard, Pricing a segmented market subject to congestion, in Proceedings of the Fifth Triennal Symposium on Transportation Analysis (TRISTAN V), Le Gosier, Guadeloupe, June 13-18 (2004).

[14] F. Gilbert, P. Marcotte and G. Savard, Global and local approaches to a logit-based pricing problem, in 2006 Optimization Days, Montreal, May 8-10 (2006).

[15] A. Grigoriev, S. van Hoesel, A. van der Kraaij, M. Uetz and M. Bouhtou, Pricing network edges to cross a river, in Approximation and online algorithms, edited by G. Persiano and R. Solis-Oba, Lect. Notes Comput. Sci. 3351, Springer-Verlag, Berlin, Heidelberg (2004) 140-153.

[16] C.G. Justus, W.R. Hargraves, A. Mikhail and D. Graber, Methods for estimating wind speed frequency distributions. J. Appl. Meteorol. 17 (1978) 350-353.

[17] N. Keon and G. Anandalingam, Optimal pricing for multiple services in telecommunications networks offering quality-of-service guarantees. IEEE/ACM Transactions on Networking 11 (2003) 66-80.

[18] N. Keon and G. Anandalingam, A new pricing model for competitive telecommunications services using congestion discounts. INFORMS J. Comput. 17 (2005) 248-262.

[19] M. Labbé, P. Marcotte and G. Savard, A bilevel model of taxation and its application to optimal highway pricing. Manage. Sci. 44 (1998) 1608-1622.

[20] M. Labbé, P. Marcotte and G. Savard, On a class of bilevel programs, in Nonlinear optimization and related topics, edited by G.D. Pillo and F. Giannessi, Kluwer Academic Publishers, Dordrecht, Boston (1999) 183-206.

[21] P. Marcotte, G. Savard and D. Zhu, Mathematical structure of a bilevel strategic pricing model. Eur. J. Oper. Res. (2006), (to appear).

[22] P. Marcotte and D. Zhu, Equilibria with infinitely many differentiated classes of customers. In Complementarity and variational problems. State of the art, edited by J.-S. Pang and M. Ferris, SIAM Publications, Philadelphia (1997) 234-258.

[23] J.I. McGill and G.J. van Ryzin, Revenue management: Research overview and prospects. Transportation Science 33 (1999) 233-256.

[24] S.K. Nair and R. Bapna, An application of yield management for internet service providers. Nav. Res. Logist. 48 (2001) 348-362.

[25] P. Reichl, D. Hausheer and B. Stiller, The cumulus pricing model as an adaptive framework for feasible, efficient and user-friendly tariffing of internet services. Comput. Networks 43 (2003) 3-24.

[26] R. Savickas, A simple option-pricing formula. Financ. Rev. 37 (2002) 207-226.

[27] A. Wächter and L.T. Biegler, On the implementation of a primal-dual interior point filter line search algorithm for large-scale nonlinear programming. Math. Program. 106 (2006) $25-57$. 